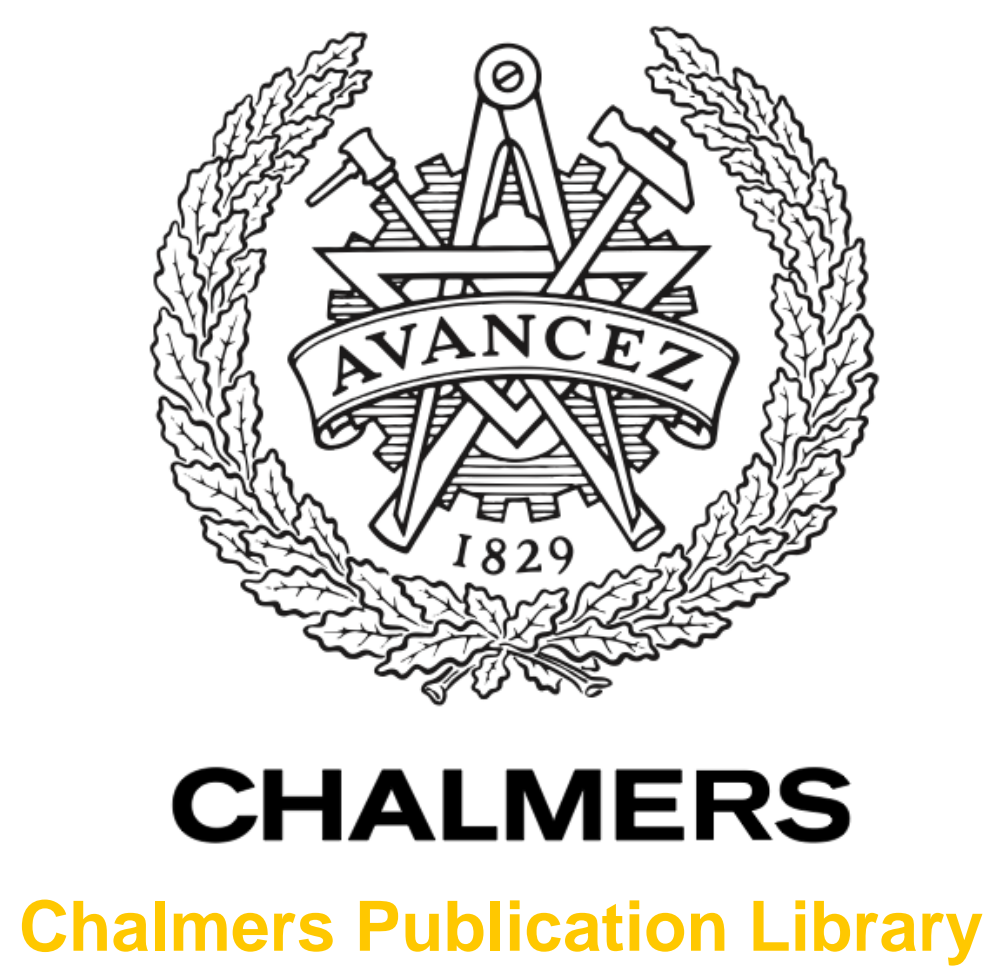

\author{
Design and evaluation of a microfluidic system for inhibition studies of yeast cell \\ signaling
}

This document has been downloaded from Chalmers Publication Library $(\mathrm{CPL})$. It is the author's version of a work that was accepted for publication in:

Proceedings of SPIE - The International Society for Optical Engineering. Optical Trapping and Optical Micromanipulation IX, San Diego, 12-16 August 2012 (ISSN: 0277-786X)

Citation for the published paper:

Hamngren Blomqvist, C. ; Dinér, P. ; Grøtli, M. (2012) "Design and evaluation of a microfluidic system for inhibition studies of yeast cell signaling". Proceedings of SPIE - The International Society for Optical Engineering. Optical Trapping and Optical Micromanipulation IX, San Diego, 12-16 August 2012

http://dx.doi.org/10.1117/12.929728

Downloaded from: http://publications.lib.chalmers.se/publication/172029

Notice: Changes introduced as a result of publishing processes such as copy-editing and formatting may not be reflected in this document. For a definitive version of this work, please refer to the published source. Please note that access to the published version might require a subscription. 


\title{
Design and evaluation of a microfluidic system for inhibition studies of yeast cell signaling
}

\author{
Charlotte Hamngren, ${ }^{a, b}$ Peter Dinér, ${ }^{c, d}$ Morten Grøtli, ${ }^{d}$ Mattias Goksör, ${ }^{a}$ and Caroline B. Adiels* ${ }^{a, d}$ \\ a Department of Physics, University of Gothenburg, S-412 96 Göteborg, Sweden, \\ Tel: +46 3178691 23; E-mail: caroline.adiels@physics.gu.se \\ ${ }^{\mathrm{b}}$ Current address: Department of Applied Physics, Chalmers University of Technology, \\ S-412 96 Göteborg, Sweden \\ ${ }^{c}$ Current address: Department of Biochemistry \& Organic Chemistry, Box 576, \\ Uppsala University, S-751 23 Uppsala, Sweden. \\ ${ }^{\mathrm{d}}$ Department of Chemistry and Molecular Biology, University of Gothenburg, \\ S-413 90 Göteborg, Sweden
}

\begin{abstract}
In cell signaling, different perturbations lead to different responses and using traditional biological techniques that result in averaged data may obscure important cell-to-cell variations. The aim of this study was to develop and evaluate a fourinlet microfluidic system that enables single-cell analysis by investigating the effect on Hog1 localization post a selective Hog1 inhibitor treatment during osmotic stress.

Optical tweezers was used to position yeast cells in an array of desired size and density inside the microfluidic system. By changing the flow rates through the inlet channels, controlled and rapid introduction of two different perturbations over the cell array was enabled. The placement of the cells was determined by diffusion rates flow simulations. The system was evaluated by monitoring the subcellular localization of a fluorescently tagged kinase of the yeast "High Osmolarity Glycerol" (HOG) pathway, Hog1-GFP. By sequential treatment of the yeast cells with a selective Hog1 kinase inhibitor and sorbitol, the subcellular localization of Hog1-GFP was analysed on a single-cell level.

The results showed impaired Hog1-GFP nuclear localization, providing evidence of a congenial design. The setup made it possible to remove and add an agent within 2 seconds, which is valuable for investigating the dynamic signal transduction pathways and cannot be done using traditional methods. We are confident that the features of the four-inlet microfluidic system will be a valuable tool and hence contribute significantly to unravel the mechanisms of the HOG pathway and similar dynamic signal transduction pathways.
\end{abstract}

Keywords: Microfluidics, single-cell analysis, signal transduction pathways, optical manipulation,

\section{INTRODUCTION}

The study of biological signaling pathways is complicated and model organisms are often used in order to explore their complexity. Saccharomyces cerevisiae (S. cerevisiae), or budding yeast, is a frequently used model organism for eukaryote cells ${ }^{1-3}$ in which biological mechanisms that could have future impact on human disease prevention are studied. ${ }^{4}$ The High Osmolarity Glycerol (HOG) pathway is one of the highly conserved Mitogen Activated Protein

Optical Trapping and Optical Micromanipulation IX, edited by Kishan Dholakia, Gabriel C. Spalding, Proc. of SPIE Vol. 8458, 84582K · C 2012 SPIE · CCC code: 0277-786/12/\$18 · doi: 10.1117/12.929728 
Kinase (MAPK) signaling pathways present in yeast. It is involved in the cellular adaptation to hyperosmotic stress and hence a good model for studying osmotic adaptation processes. ${ }^{5}$ The MAPK Hog1 is activated by changes in external osmolarity. The activation signal is transduced via two branches which merge by the phosphorylation of the MAPK kinase (MAPKK) Pbs2. Pbs2 subsequently phosphorylates and activates the MAPK Hog1, which rapidly translocates from the cytoplasm into the nucleus. ${ }^{6}$

Studying biological processes such as dynamic signal transduction pathways with traditional biological techniques will result in averaged data. Single-cell analysis however, provides information on cell-to-cell variability obscured in averaged results from population analyses ${ }^{7}$ that might be valuable for the understanding of the mechanisms. Microfluidics can effectively be used for single cell studies and result in fast and reproducible introduction of different perturbations on the studied system. Moreover, the spatial and temporal resolution of such analyses are widely exceeding that of traditional cell culture methodologies. ${ }^{8}$

In this work we have designed and evaluated a four-inlet microfluidic system in order to study the time-resolved translocation process of Hog 1 upon treatment with increased osmolarity in the presence of a Hog1-specific inhibitor. Cells were positioned with optical tweezers $(\mathrm{OT})^{9}$ and the setup made it possible to accomplish cell milieu change within $2 \mathrm{s.}{ }^{10}$ Evaluation was performed by perturbing the yeast cells with the Hog1 inhibitor 4-(1-benzyl-4-phenyl-1H-1,2,3triazol-5-yl)-N-isopropylpyridin-2-amine ${ }^{11}$ and subsequently with sorbitol, inducing hyperosmotic stress. The cells were imaged with axial stack fluorescence microscopy, capturing the by the osmotic stress initiated nuclear migration of the Hog1-GFP reporter protein.

\section{METHODOLOGY}

\subsection{Yeast strain and culturing}

Hog1 migration was monitored by the reporter protein Hog1-GFP. The yeast strain used was HOG1-GFP-HIS3 NRD1mCherry-hphNT1 in BY4741 background. This strain contained also NRD1-mCherry that enabled nuclei localization throughout the time lapse experiment by its nucleus resident reporter protein expression. The cells were grown in Yeast Nitrogen Base (YNB, $6.7 \mathrm{~g} / \mathrm{l})$ with Complete Supplement Mixture (CSM, $1.54 \mathrm{~g} / \mathrm{l})$ and 2\% Glucose $(\mathrm{pH}=6)$, at $30{ }^{\circ} \mathrm{C}$ on a shaker $(220 \mathrm{rpm})$. The cells were collected at $\mathrm{OD}_{600}=0.5-1.0$, and concentrated by $30 \mathrm{~s}$ spinning in a tabletop centrifuge at $6000 \mathrm{rpm}$. A sorbitol concentration of $500 \mathrm{mM}$ was used for exposing the cells to osmotic shock.

\subsection{Inhibitor}

The selective Hog1 inhibitor 4-(1-benzyl-4-phenyl-1H-1,2,3-triazol-5-yl)-N-isopropylpyridin-2-amine ${ }^{11}(\mathrm{M}=478.67$ $\mathrm{g} / \mathrm{mol}$ ) was dissolved in dimethyl sulfoxide (DMSO) to a suitable stock solution. The final concentrations range between $10 \mathrm{nM}$ to $25 \mu \mathrm{M}$ and control experiments contained the corresponding maximum volume of DMSO. The approximated diffusion coefficient was determined to $260 \mu \mathrm{m}^{2} / \mathrm{s}$ (measured at $22{ }^{\circ} \mathrm{C}$ ). The Hog1 inhibitor incubation time was set to 20 $\min$ and a sorbitol-inhibitor mixture was introduced at $\mathrm{t}=0$. Using a sorbitol-inhibitor mixture instead of pure sorbitol will induce osmotic pressure without removing the inhibitory signal.

\subsection{Microfluidic fabrication and assembly}

The design of the microfluidic systems was delineated and simulated using the software COMSOL Multiphysics; Chemical Engineering module. The concentration distributions of the sorbitol and inhibitor respectively as well as the velocity field within the device were simulated using the application modes Incompressible Navier-Stokes and Convection and Diffusion.

The manufacturing process and experimental procedure have previously been reported. ${ }^{10,12}$ The disposable microfluidic systems were made from poly(dimethylsiloxane) (PDMS) (Sylgard184, Dow Corning) using soft lithography and sealed off with a cover glass. A reusable master relief pattern was created on a silicon wafer and PDMS was poured onto it using a formwork containing pins to form the four inlet holes and the outlet of the microfluidic system. After polymerization, each inlet channel was $27 \mu \mathrm{m}$ high and $100 \mu \mathrm{m}$ wide. To form a strong irreversible seal, the PDMS 
structure and a glass slide were treated with air plasma and thereafter immediately placed in physical contact with each other. $^{13}$

The finalized system was treated with the lectin protein concanavalin A to saturate the inner surfaces of the system. Concanavalin A promotes adhesion between the cells and the surfaces by binding to sugar structures on the yeast cells. ${ }^{14}$ Just prior the experiment, glass syringes (Hamilton Company) were attached to the microfluidic system via polytetrafluoroethylene tubing, containing the cells and different media respectively (Figure 1).

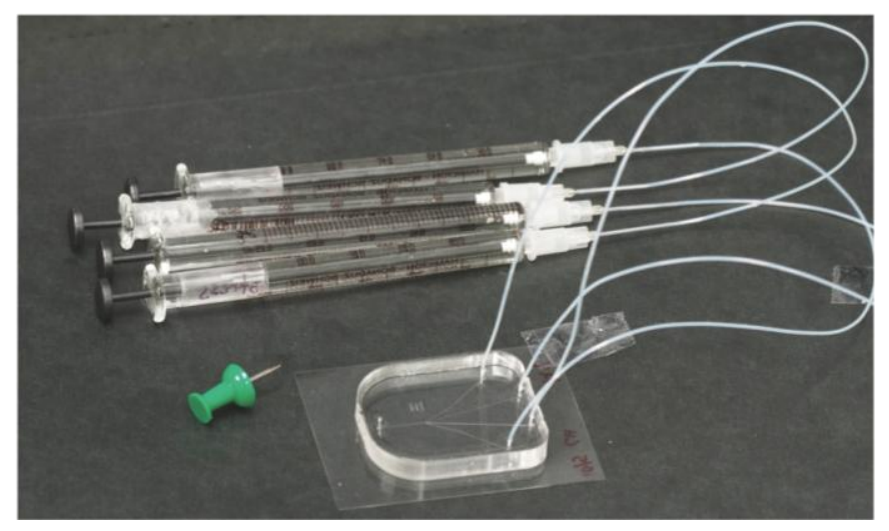

Figure 1. The microfluidic setup where syringes filled with cells and substances are connected to the four inlet system channels. Size compared to a thumbtack.

Micro-environmental changes necessary for monitoring the Hog1-GFP migration, was created by changing the flow rates in the inlet channels set by syringe pumps (CMA 400, CMA Microanalysis). In this way, the environment could rapidly be changed from neutral media to a media containing Hog1 inhibitor (for inhibition of Hog1), or sorbitol (for inducing osmotic stress) or a mixture thereof. The automation software OpenLab (Perkin Elmer), used for controlling the syringe pumps, the microscope and the EM-CCD camera (C9100-12, Hamamatsu Photonics) used for the image capturing, enabled a completely automated setup and maximized the control of the experimental process.

\subsection{Forming a cell array using optical tweezers}

A thorough explanation of the optical setup is to be found in an accompanying proceeding. ${ }^{15}$ Using a fiber-coupled ytterbium laser (YLD-5-LP, IPG Photonics) with a wavelength of $1070 \mathrm{~nm}$, single cells were actively chosen, caught and moved from the flow of the cell inlet channel in a non-intrusive way. ${ }^{10}$ One at a time, cells were then gently pressed against the bottom of the system causing them to adhere. By repeating this action several times, an array of cells was formed on the system floor. This trapping event rendered the cells exposed to the infrared light only for a few seconds and ensured minimized photodamage. ${ }^{10}$

\subsection{Imaging and analysis}

The imaging time interval was $30 \mathrm{~s}$ during the first five minutes of the experiment and less frequent as the experiment progressed in order to capture the Hog1 dynamics while minimizing the bleaching of the fluorophores. The acquiring of the images was performed using the highest level of the attenuator of the $15 \mathrm{~W}$ mercury short-arc reflector lamp (EL6000, Leica Microsystem) with an exposure time of $300 \mathrm{~ms}$. This in order for the fluorescent intensity results to be comparable with earlier published single-cell measurements. ${ }^{10}$ The time series imaging started at $\mathrm{t}=-30 \mathrm{~s}$ and images from 29 time points were collected. For each time point, an axial stack of seven optical sections with an internal distance of $0.8 \mu \mathrm{m}$ was acquired for bright field, GFP and mCherry respectively, see Figure 2.

Every image sequence contained 21 photos ( $3 \times 7$ photos) per time point, and the maximum imaging frequency was two image sequences per minute. In total, 609 images were analyzed for each experiment. The fluorescence images showed the Hog1-GFP and Nrd1-mCherry intensities in every time point. In order to interpret the migration of Hog1-GFP, the 
exact locations of the cell wall and the nuclear membrane had to be known. This information was retrieved from the outline of the cell contours from a bright field image and the contours of the Nrd1-mCherry intensity (the fluorescently marked protein restricted to the nucleus) respectively.

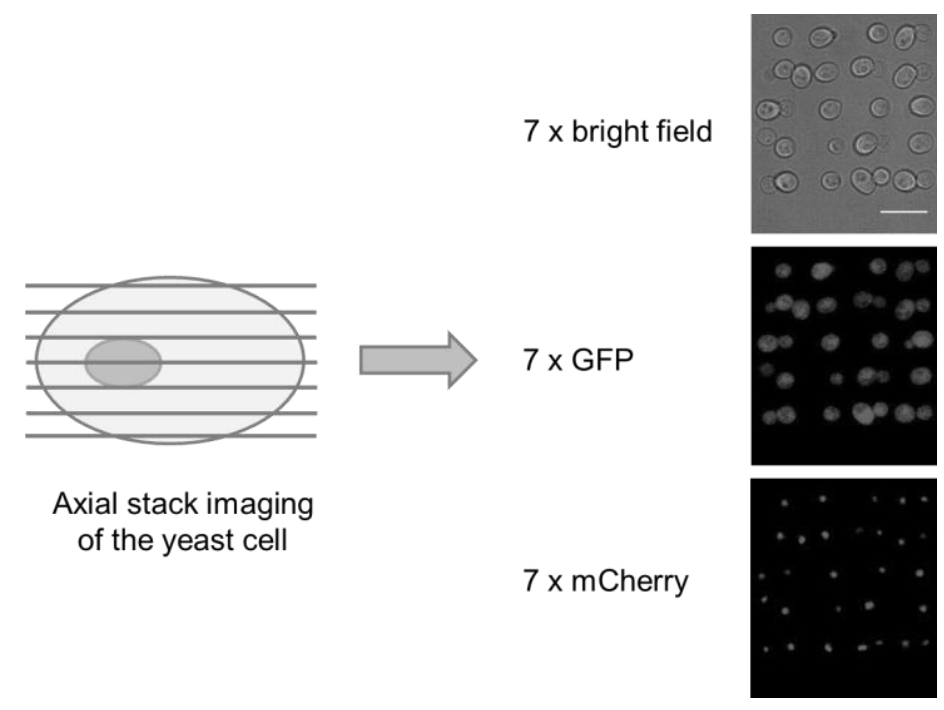

Figure 2. A schematic showing the imaging of the yeast cells in every time point. The imaging starts at -30 seconds, before the sorbitol is added, but 20 minutes post inhibitor incubation. The cells are imaged for 45 minutes at 29 time points in bright field, GFP and mCherry respectively. The automation captures an axial stack of seven optical sections with each light setting resulting in over 600 images for image analysis, which is done with the software Cellstress. The scale bar is $10 \mu \mathrm{m}$.

The GFP fluorescence intensity was used for the single-cell analysis by Cellstress ${ }^{16}$, as a measure of the number of Hog1 proteins in a subcellular location. The most fluorescently intense optical section for each individual cell was automatically identified and the GFP fluorescence images and the bright field images were used for calculating the ratio (R) between the mean intensities from Hog1-GFP in the nucleus and the cytosol respectively;

$$
R=\frac{\text { Mean intensity in the nucleus }}{\text { Mean intensity in the cytosol }} \propto \frac{\left[\text { Hog } 1_{\text {nucleus }}\right]}{\left[\text { Hog } 1_{\text {cytosol }}\right]} .
$$

\section{RESULTS}

\subsection{The concentration gradients and the velocity profiles}

Simulations of three different flow configurations of sorbitol and Hog1 inhibitor were performed for the four-inlet microfluidic system, respectively (Figure 3A):

I. The configuration used when trapping cells with the OT and placing them in an array required flow rates of $80 \mathrm{nl} / \mathrm{min}$ in the inlet channels containing the cells and neutral medium and $40 \mathrm{nl} / \mathrm{min}$ in the inlet channels containing inhibitor and stress.

II. The configuration for inhibitor incubation required flow rates of $40 \mathrm{nl} / \mathrm{min}$ in the cell, neutral and stress channel respectively and $400 \mathrm{nl} / \mathrm{min}$ in the inhibitor channel.

III. The configuration for stress treatment required flow rates of $40 \mathrm{nl} / \mathrm{min}$ in all inlet channels except the stress channel, which required $800 \mathrm{nl} / \mathrm{min}$.

The cell array was positioned to be exposed to a specific concentration from only one inlet channel at a time (e.g. culture medium, inhibitor solution or the sorbitol-inhibitor mixture) and its location (Figure 3B) was selected based on this simulated data. 
A

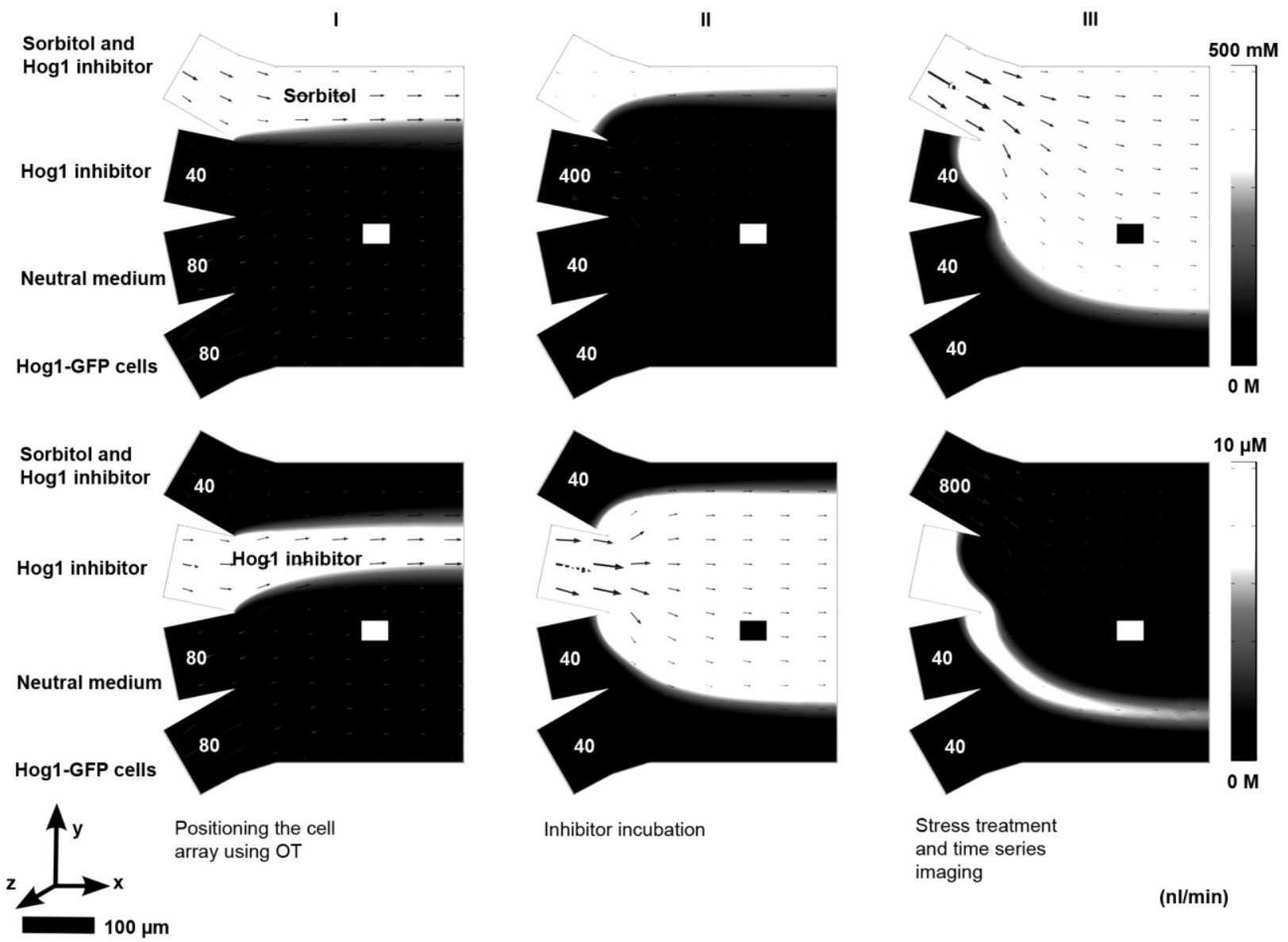

B

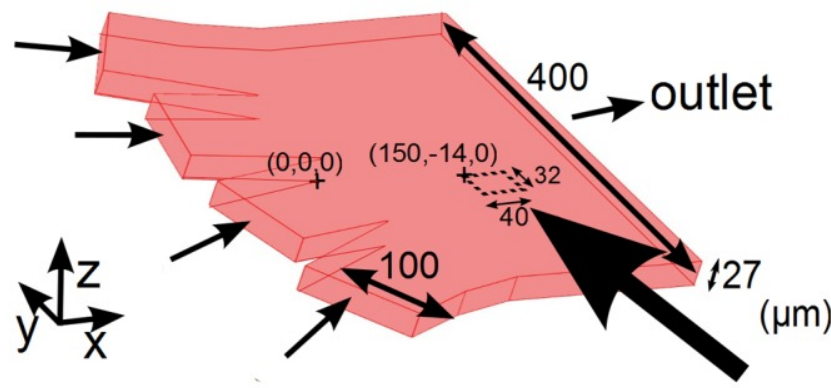

Figure 3. Properties of the cell array in the four-inlet microfluidic system. (A) Simulations of typical flows and perturbation concentrations. The arrow length is proportional to the flow velocity (given at a distance of $13.5 \mu \mathrm{m}$, half of the channel height, from the bottom surface). The concentration distribution is given at a distance of $0.1 \mu \mathrm{m}$ from the bottom surface. The rectangles represent the position of the yeast cells and the pump rates are shown in $\mathrm{nl} / \mathrm{min}$ for each inlet channel. The shaded gradient represents the sorbitol flow from 0 to $500 \mathrm{mM}$ (upper row) and the inhibitor flow from 0 to $10 \mu \mathrm{M}$ (lower row) respectively. The first configuration (I) is used for positioning the cell array using optical tweezers, the second configuration (II) is used for inhibitor incubation and the third configuration (III) is used for sorbitol treatment and imaging. (B) Shows the channel junction and the cell array site (large arrow). The coordinate system (x, y, z) of the microfluidic system has its origin at the junction between the two middle inlet channels. The array is placed with the upper most left cell at $(150,-14,0)$. 


\subsection{The Hog1 inhibitor impairs Hog1 nuclear localization using the four-inlet microfluidic system}

In order to determine how efficient the inhibitor enters the yeast cells under flow conditions, a yeast cell array was exposed to the Hog1 inhibitor within the four-inlet microfluidic system.

Migration studies upon Hog1 pathway activation, where the intensity ratio of nucleic to cytosolic Hog1-GFP was plotted revealed that the Hog1 inhibitor is taken up by the cells when present in a flow and impairs Hog1 nuclear localization (Figure 4). The single-cell data in Figure 4, clearly demonstrates uptake of the inhibitor into the cells when present in a flow. When the cells are treated with a $25 \mu \mathrm{M}$ Hog1 inhibitor 4-(1-benzyl-4-phenyl-1H-1,2,3-triazol-5-yl)-Nisopropylpyridin-2-amine and subsequently a sorbitol-inhibitor mixture in the four-inlet microfluidic system, their Hog1GFP nuclear localization response is impaired compared to the response in sorbitol only treated cells. This is in agreement with previous results obtained with the Hog1-inhibitor ${ }^{11}$ and also provides evidence for a congenial system design.

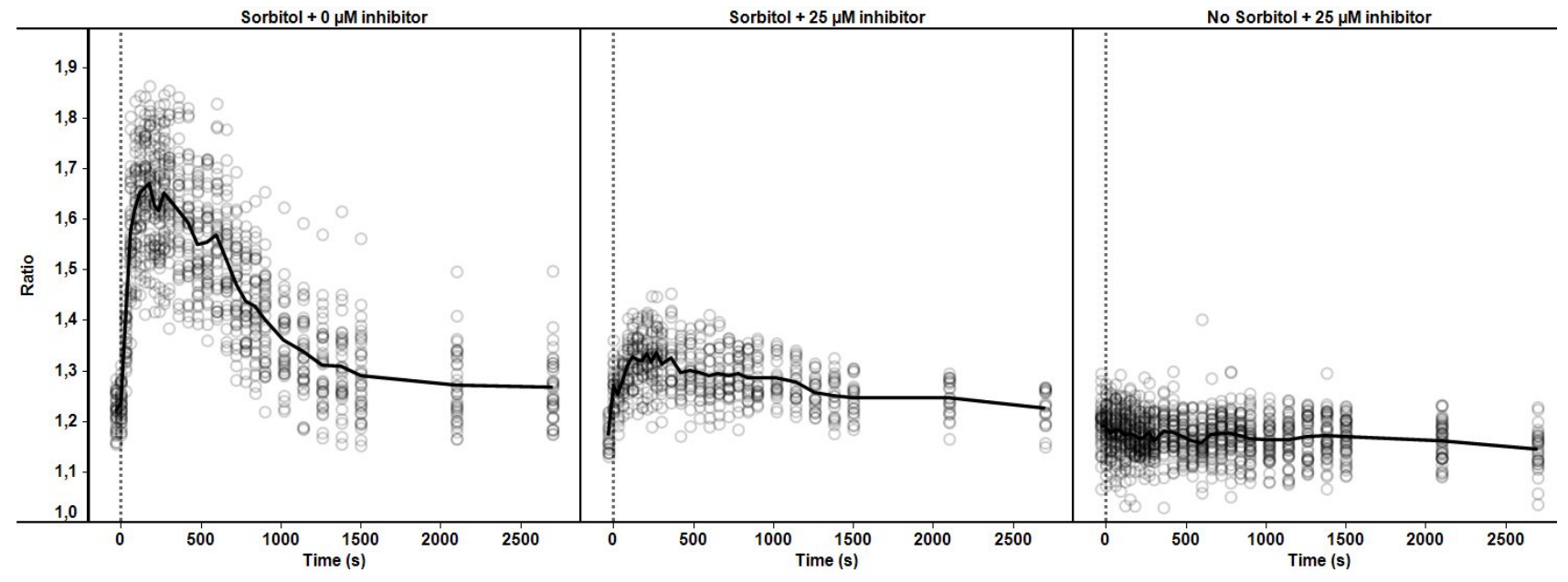

Figure 4. A single-cell analysis presentation of the selective Hog1-inhibitor treatment using the four-inlet microfluidic system. In the left panel, single-cell responses (dots) at each time point represent the increased nuclear Hog1-GFP localization upon sorbitol addition without prior inhibitor incubation. The middle panel shows the corresponding single-cell responses when the cells have been treated with the inhibitor. The rightmost panel shows the cellular variation over time without stress perturbation, only inhibitor incubation. At the indicated inhibitor concentration of $25 \mu \mathrm{M}$, it is clearly shown that the Hog1-GFP nuclear migration is impaired compared to sorbitol treatment only. The black line corresponds to the mean ratio values for the whole cell array. Data were acquired from $t=-30 \mathrm{~s}$ to $\mathrm{t}=2700 \mathrm{~s}$ and the sorbitol-inhibitor mixture $(500 \mathrm{mM}$ sorbitol) was introduced into the microfluidic system at $\mathrm{t}=0 \mathrm{~s}$.

\section{CONCLUSIONS}

In conclusion, a microfluidic device with four inlet channels has, in an optical tweezers setup, been used for multiple perturbation treatments of a yeast signal transduction pathway and for studying the subsequent dynamic single-cell responses. Not only is the spatial resolution of this setup on a sub cellular basis, but the temporal resolution is controlled on a level of seconds which results in high quality data.

The automatic setup enables low inter-experimental variation and the custom made automations will provide the opportunities to elaborate with a removal of either perturbation during measurement. This would be extremely hard - not to say impossible - to accomplish using traditional methods of biology.

We are confident that the features of the four-inlet microfluidic system will be a valuable tool and hence contribute significantly to unravel the mechanisms of the HOG and similar dynamic signal transduction pathways. 


\section{ACKNOWLEDGEMENTS}

We would like to thank Fraunhofer Chalmers Research Centre Industrial Mathematics for providing the Cellstat software, the department of Cell and Molecular Biology for yeast strains, the Swedish NMR centre for the diffusion coefficient measurements and Martin Adiels for Tableau support.

This work was supported by the Swedish Research Council (VR), Carl Trygger Foundation for Scientific Research and the European Commission programs AMPKIN and UNICELLSYS. The microfluidic devices were developed at the research facility Centre for Biophysical Imaging, sponsored by the University of Gothenburg.

\section{REFERENCES}

[1] J. C. Mell, and S. M. Burgess, [Yeast as a Model Genetic Organism] John Wiley \& Sons, Ltd, (2001).

[2] D. Botstein, S. A. Chervitz, and J. M. Cherry, "Genetics - Yeast as a model organism," Science, 277(5330), 1259-1260 (1997).

[3] W. H. Mager, and J. Winderickx, "Yeast as a model for medical and medicinal research," Trends in Pharmacological Sciences, 26(5), 265-273 (2005).

[4] L. H. Hartwell, "Yeast and cancer," Bioscience Reports, 24(4-5), 523-544 (2004).

[5] E. De Nadal, F. X. Real, and F. Posas, "Mucins, osmosensors in eukaryotic cells?," Trends in Cell Biology, 17(12), 571-574 (2007).

[6] E. Klipp, B. Nordlander, R. Kruger et al., "Integrative model of the response of yeast to osmotic shock," Nat Biotechnol, 23(8), 975-82 (2005).

[7] D. Di Carlo, and L. P. Lee, "Dynamic single-cell analysis for quantitative biology," Analytical Chemistry, 78(23), 7918-7925 (2006).

[8] H. A. Svahn, and A. van den Berg, "Single cells or large populations?," Lab on a Chip, 7(5), 544-546 (2007).

[9] A. Ashkin, J. M. Dziedzic, and T. Yamane, "Optical trapping and manipulation of single cells using infrared laser beams," Nature, 330(6150), 769-71 (1987).

[10] E. Eriksson, K. Sott, F. Lundqvist et al., "A microfluidic device for reversible environmental changes around single cells using optical tweezers for cell selection and positioning," Lab on a Chip, 10(5), 617-625 (2010).

[11] P. Dinér, J. Veide Vilg, J. Kjellén et al., "Design, Synthesis, and Characterization of a Highly Effective Hog1 Inhibitor: A Powerful Tool for Analyzing MAP Kinase Signaling in Yeast," PLoS ONE, 6(5), e20012 (2011).

[12] K. Sott, E. Eriksson, and M. Goksör, [Biomolecular Separation and Analysis] Caister Academic Press, Norwich(2009).

[13] D. C. Duffy, J. C. McDonald, O. J. A. Schueller et al., "Rapid prototyping of microfluidic systems in poly(dimethylsiloxane)," Analytical Chemistry, 70(23), 4974-4984 (1998).

[14] D. Mislovicova, J. Masarova, J. Svitel et al., "Influence of mannan epitopes in glycoproteins - Concanavalin A interaction. Comparison of natural and synthetic glycosylated proteins," International Journal of Biological Macromolecules, 30(5), 251-258 (2002).

[15] A.-K. Gustavsson, B. C. Adiels, and M. Goksör, "Induction of sustained glycolytic oscillations in single yeast cells using microfluidics and optical tweezers," Proc. SPIE 8458-34, (2012).

[16] M. Smedh, C. Beck, K. Sott et al., "CellStress - open source image analysis program for single-cell analysis," Proc. SPIE, 7762,, 77622N-77622N-11 (2010). 